\title{
Marc Loriol, La construction du social. Souffrance, travail et catégorisation des usagers dans l'action publique
}

Rennes, Presses universitaires de Rennes, coll. « Le sens social », 2012

\section{Arnaud Mias}

\section{OpenEdition}

Journals

Édition électronique

URL : http://journals.openedition.org/travailemploi/6279

DOI : 10.4000/travailemploi.6279

ISSN : 1775-416X

Éditeur

DARES - Ministère du Travail

Édition imprimée

Date de publication : 15 mars 2014

Pagination : 143-144

ISSN : 0224-4365

\section{Référence électronique}

Arnaud Mias, « Marc Loriol, La construction du social. Souffrance, travail et catégorisation des usagers dans l'action publique ", Travail et Emploi [En ligne], 137 | janvier-mars 2014, mis en ligne le 01 janvier 2014, consulté le 22 septembre 2020. URL : http://journals.openedition.org/travailemploi/6279 ; DOI : https://doi.org/10.4000/travailemploi.6279

Ce document a été généré automatiquement le 22 septembre 2020

(c) Direction de l'animation de la recherche, des études et des statistiques (Dares) 


\title{
Marc Loriol, La construction du social. Souffrance, travail et catégorisation des usagers dans l'action publique
}

Rennes, Presses universitaires de Rennes, coll. « Le sens social », 2012

\author{
Arnaud Mias
}

\section{RÉFÉRENCE}

Marc Loriol, La construction du social. Souffrance, travail et catégorisation des usagers dans l'action publique, Rennes, Presses universitaires de Rennes, coll. « Le sens social », 2012, $214 \mathrm{p}$.

1 Dans cet ouvrage issu de son mémoire d'habilitation à diriger des recherches, Marc Loriol propose une réflexion de synthèse à partir des travaux qu'il a menés depuis vingt ans sur l'influence des milieux de travail dans la "construction du social ». Cette construction se manifeste notamment à travers la genèse d'entités cliniques en santé mentale et leur usage privilégié par certains salariés pour qualifier leur mal-être au travail. Elle fonde aussi la manière dont des fonctionnaires catégorisent les usagers qu'ils ont en face d'eux pour réaliser leur activité. Dans les deux cas, qui nourrissent les développements des chapitres 2 et 3 de l'ouvrage, Marc Loriol révèle un même travail de mise en forme de l'expérience des contraintes vécues dans l'activité et de la perception des situations de travail, qui soutient la gestion collective des difficultés rencontrées et tend à faire coïncider les pratiques quotidiennes avec les conceptions plus ou moins idéalisées que les individus se font de leur activité professionnelle.

2 En appui de ces développements, un premier chapitre trace «Les espaces du constructivisme" et revient sur les principales controverses que cette approche suscite. Marc Loriol montre clairement la distance qui sépare sa démarche du relativisme. Plutôt que d'affirmer que le monde, matériel comme social, n'existe qu'à travers les représentations que les individus s'en font, le constructivisme interroge les 
liens complexes, parce que circulaires, qu'entretiennent les deux ordres de réalité : si les faits objectifs contraignent les représentations et les connaissances qui s'y rapportent, le sens subjectif qui leur est attribué contribue à les altérer, à les transformer. Ce sens est le produit de processus collectifs, de schèmes, de façons de voir qui résultent eux-mêmes de constructions sociales passées, stabilisées voire cristallisées et qui agissent telles des institutions, contraignant les pensées, actions et interactions des individus. C'est ce caractère éminemment processuel et multiniveaux qui rend le social si complexe à appréhender, et qui justifie en même temps l'adoption d'une posture de recherche relevant d'un constructivisme modéré et pragmatique. Car pour Marc Loriol, l'enjeu est d'abord d'adopter la perspective méthodologique la plus efficace pour comprendre un monde social qui fonctionne comme un «ensemble de constructions sociales, plus ou moins achevées, plus ou moins abouties (donc plus ou moins contraignantes pour les individus)» (p. 34). Cela rend décisive la définition de l'objet de la recherche : dans cet ensemble inachevé et évolutif, que va-t-on considérer comme acquis ou stable, que l'on va donc se contenter d'appréhender comme un contexte contraignant? Sur quel phénomène va-t-on concentrer l'analyse pour en retracer au contraire la genèse ? Cette définition de l'objet d'étude conduit à limiter la démarche à une des multiples perspectives constructivistes disponibles.

C'est ici que réside certainement l'un des apports fondamentaux de l'ouvrage de Marc Loriol à la théorie sociologique. Partant d'une "mosaïque " constructiviste, l'auteur circonscrit progressivement l'espace d'analyse à quatre perspectives : l'action collective à travers le "claim making process", l'élaboration d'une cause, d'un enjeu collectif qui sert de support aux mobilisations collectives; la définition de la situation; l'étiquetage et la catégorisation ; la carrière et la trajectoire. Cette typologie permet de souligner les articulations plutôt que l'opposition entre ces différentes approches constructivistes. Elle sert surtout à confirmer le pragmatisme de la méthodologie défendue : «suivant que la "construction" est plus ou moins le résultat d'un mouvement social conscient ou organisé ou le produit conflictuel de la confrontation de "définitions de la situation" divergentes, les analyses en termes de construction des problèmes publics ou de "définition de la situation" seront plus ou moins appropriées» (pp. 57-58). On perçoit d'ailleurs des articulations plus fortes entre certaines perspectives : « le moment clé de cette "carrière", l'étiquetage, le diagnostic du patient comme buveur pathologique peut être l'objet d'une étude en soi. En fait, la carrière est jalonnée par des moments d'évaluation, de qualification de la personne» (p.72). Cela étant, comme ces perspectives privilégient des méthodes d'enquête distinctes (corpus documentaire conséquent, entretiens, observation in situ ou récits de vie), un programme de recherche qui entendrait les embrasser toutes ensemble semble hors d'atteinte.

Le chapitre 2 articule, autour de la question des risques psychosociaux, deux de ces perspectives, la définition de la situation et le "claim making process ». Il s'agit d'abord de comprendre comment on produit du sens sur des situations de travail: "Les différents acteurs sociaux concernés par le mal-être au travail produisent, adaptent, transforment ou rejettent les différentes entités cliniques pour donner du sens à la situation à laquelle ils sont confrontés et qu'ils contribuent ainsi à faire évoluer " (p. 75). En sens inverse, Marc Loriol retrace également la genèse de différentes entités cliniques (syndrôme de fatigue chronique, stress, burn out), en portant une attention particulière au moment décisif où une nouvelle catégorie est ou non authentifiée, démontrée, justifiée, qui permet alors de proposer une nouvelle définition de la réalité. 
Selon les catégories, différentes tactiques de validation scientifique et sociale ont été employées, sur lesquelles l'auteur fait porter l'analyse.

5 Un même mouvement dialectique anime le troisième chapitre, consacré au travail relationnel développé par les agents publics en contact avec les «usagers» des politiques publiques ("street-level bureaucrats»). Marc Loriol montre que, pour exécuter leur travail de la façon la plus proche de la conception idéale qu'ils se font de leur activité, les salariés reconstruisent l'usager, le mettent en forme à partir de catégories de pensée qui fonctionnent comme des routines, orientent le «traitement» de la situation, restreignent le champ des activités professionnelles autorisées, des tâches légitimes, et se protègent ainsi de toute remise en cause de leur identité professionnelle. Deux groupes professionnels sont étudiés : les infirmières hospitalières et les policiers de voie publique, aux prises avec leurs «bons » malades et leurs « vrais clients »-délinquants. Les mécanismes de catégorisation des usagers, accomplis par ces "street-level bureaucrats", exercent un effet en retour sur les politiques publiques, influençant très directement leur mise en œuvre. Marc Loriol prolonge alors sa réflexion en analysant comment décideurs et praticiens ont fait des «jeunes » et des " vieux » des catégories à risque, cadrant le parcours et les expériences des personnes ainsi qualifiées. Là encore, la circularité de la construction du social se donne à voir, les réactions des usagers conduisant elles-mêmes à des ajustements, à des réorientations de ces politiques publiques. Elle se manifeste plus largement dans l'influence réciproque entre groupes professionnels et politiques publiques: si les premiers influencent la mise en œuvre et la conception des secondes, celles-ci façonnent en retour la morphologie et l'identité de ceux-là.

Dans cet ouvrage, Marc Loriol propose une construction théorique magistrale tout en étant très pédagogique dans son déploiement. Il réussit à rendre limpides des perspectives complexes et souvent audacieuses. Certes, certains passages paraissent plus stimulants, parce que plus originaux, que d'autres. Mais l'application du cadre analytique aux différents matériaux d'enquête traités est tout à fait convaincante et constitue un apport à la connaissance des métiers de la fonction publique. Au final, loin de tout subjectivisme, l'auteur rappelle la centralité des collectifs de travail dans l'activité professionnelle, collectifs qui viennent appuyer un jugement, une définition de la situation, une catégorisation ou un cadrage de l'interaction. Par extension, le rôle des syndicats dans cette construction du social est à plusieurs reprises souligné. Si ces collectifs sont si importants, c'est que les situations de travail se caractérisent par une profonde ambivalence. Le sens qui leur est donné, les façons de les vivre sont multiples et largement dépendantes de la définition intersubjective que les acteurs en donnent.

7 L'ouvrage représente une balise précieuse pour explorer avec recul et intelligibilité une «mosaïque constructiviste» qui peut donner l'impression de couvrir un territoire extrêmement large, voire dominant, des sciences sociales. D'où l'intérêt de la typologie qui propose des définitions limitées et pragmatiques - parce qu'inspirées d'une démarche de recherche empirique - des traditions constructivistes. Outre qu'il est dommage, vu l'ambition du propos, que plusieurs contributions évoquées dans ce tableau général aient été omises dans les références indiquées en bibliographie, on peut relever certains angles morts de l'impressionnante revue de littérature proposée. L'économie des conventions, et notamment la contribution d'Alain Desrosières, n'est ainsi pas située dans l'ensemble. Aucune référence n'est faite non plus à la théorie de la régulation sociale de Jean-Daniel Reynaud, alors même qu'est au cœur de cette 
approche l'idée d'une construction sociale contingente, résultat d'un travail collectif. Ce n'est pas un des moindres mérites de l'ouvrage que d'inciter ainsi son lecteur à reconsidérer le positionnement et les apports d'autres traditions et corpus théoriques à l'aune des perspectives stimulantes tracées par son auteur.

\section{AUTEURS}

\section{ARNAUD MIAS}

Université de Rouen ; IDHES (UMR CNRS 8533, Institutions et dynamiques historiques de l'économie et de la société) 